\title{
Comparison of Treatment Strategies for Patients With Clinical Stage T1-3/N2 Lung Cancer
}

Ya-Fu Cheng, MDa; Wei-Heng Hung, MDa; Heng-Chung Chen, MDa; Ching-Yuan Cheng, MDa; Ching-Hsiung Lin, MD, PhD ${ }^{\mathrm{b}}$; Sheng-Hao Lin, MD, PhD ${ }^{\mathrm{b}}$; and Bing-Yen Wang, MD, PhDa,c,d,e,f,

\begin{abstract}
Background: The therapeutic strategies for clinical stage T1-3N2 (cT1-3N2) lung cancer are controversial. For operable tumors, treatment can vary by center, region, and continent. This study aimed to identify the optimal therapeutic method and type of surgical strategy for cT1-3N2 lung cancer. Methods: This retrospective evaluation analyzed the records of 17,954 patients with cT1-3N2 lung cancer treated in 2010 through 2015 from the SEER database. The effects of different therapeutic methods and types of surgical strategies on overall survival (OS) were assessed. Univariate and multivariate analyses were performed using a Cox proportional hazards model. Results: The 5 -year OS rates were $27.7 \%$ for patients with T1N2 disease, $21.8 \%$ for those with T2N2 disease, and $19.9 \%$ for T3N2 disease. Neoadjuvant therapy plus operation (OP) plus adjuvant therapy, and OP plus adjuvant therapy, provided better 5 -year OS rates than OP alone or concurrent chemoradiotherapy $(34.1 \%$, $37.7 \%, 29.3 \%$, and $16.1 \%$, respectively). In the T1N2, T2N2, and T3N2 groups, lobectomy provided better 5-year OS than pneumonectomy, sublobectomy, and no surgery. Both univariate and multivariate analyses showed that young age, female sex, well-differentiated histologic grade, adenocarcinoma cell type, neoadjuvant and adjuvant therapy, lobectomy, and T1 stage were statistically associated with better 5-year OS rates. Conclusions: In cT1-3N2 lung cancer, multimodal treatments tended to provide better 5-year OS than OP alone or concurrent chemoradiotherapy. In addition, lobectomy was associated with better survival than other operative methods.
\end{abstract}

J Natl Compr Canc Netw 2020;18(2):143-150 doi: 10.6004/jnccn.2019.7353

aDivision of Thoracic Surgery, Department of Surgery, and bivision of Chest Medicine, Department of Internal Medicine, Changhua Christian Hospital, Changhua; 'School of Medicine, College of Medicine, Kaohsiung Medical University, Kaohsiung; ${ }^{d}$ Institute of Genomics and Bioinformatics, and ${ }^{\text {eNational }}$

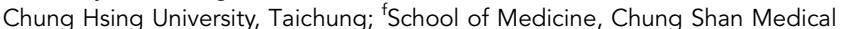
University, Taichung; and ${ }^{9}$ Center for General Education, Ming Dao University, Changhua, Taiwan.

\section{Background}

Lung cancer is the leading cause of cancer death worldwide among both men and women. ${ }^{1}$ Only $14 \%$ to $18 \%$ of all patients with lung cancer remain alive 5 years after diagnosis. ${ }^{2,3}$ The treatment strategies for stages I, II, IIIb (T4N2 and N3), and IV lung cancer have been well researched and accepted globally. However, the therapeutic methods for clinical T1-3N2 (cT1-3N2) lung cancer remain controversial and differ among centers, regions, and continents. For decades, this issue has been discussed, but no specific therapy has been accepted widely.

Therapeutic methods for cT1-3N2 lung cancer are determined through a multidisciplinary team discussion, including a board-certified thoracic surgeon, who evaluates whether the tumor is operable. ${ }^{4}$ If the tumor is inoperable, the current, widely accepted treatment is concurrent chemoradiotherapy (CCRT) followed by durvalumab. If the tumor is operable, then multimodal therapies may be used, including operation (OP) plus adjuvant chemotherapy $(\mathrm{C} / \mathrm{T})$ with or without radiotherapy (RT), and neoadjuvant $\mathrm{C} / \mathrm{T}$ or chemoradiotherapy (CRT) plus OP plus adjuvant C/T with or without RT. Rocco et $\mathrm{al}^{5}$ claimed that North American surgeons, compared with those on other continents, are less likely to offer surgical treatment and more likely to use induction therapy before resection, whereas European surgeons preferred to offer OP followed by adjuvant therapy in select cases of N2 disease.

Different therapeutic methods were compared in previous studies. ${ }^{6}$ Although many issues remain unresolved, most contemporary lung cancer studies include surgery in the multimodal treatment of cT1-3N2 disease.

This study aimed to identify the optimal therapeutic method and surgical strategy for cT1-3N2 lung cancer. We designed a retrospective study and obtained data from the SEER database, which were used to compare the overall survival (OS) rates of patients with cT1-3N2 lung cancer treated using different therapeutic methods and surgical strategies. 


\section{Methods}

\section{Patient Population and Selection}

Lung cancer cases occurring in 2010 through 2015 (classified using the 7th edition of the AJCC Cancer Staging Manual $^{\text {) }}$ and their corresponding details were obtained from the SEER public access database using SEER*Stat version 8.3.5. This study was approved by the Institutional Review Board at Changhua Christian Hospital and was deemed exempt from full review by the internal review board because the released information would be used strictly for research purposes.

An independent cohort of cases from 2016 was obtained for cutoff validation. Patients with cT1-3N2M0 lung cancer who underwent surgical resection (pneumonectomy, lobectomy/bilobectomy, or sublobectomy) or $\mathrm{C} / \mathrm{T}$, with or without RT, were eligible. Patients were excluded if they received no therapy or if their database records were missing values for therapy or type of surgery.

Baseline patient demographics (age and sex), tumor characteristics (histologic grade, cell type, $\mathrm{T}$ stage), and treatment details (surgery type, C/T, RT) were collected from the SEER database. We classified histologic subtypes as adenocarcinoma, squamous cell carcinoma (SCC), and other histologic types. Patients were divided into different cohorts based on T1N2, T2N2, and T3N2 stages. Outcome measures for our study were 1-, 3-, and 5 -year OS rates. OS was calculated as the time from date of diagnosis to either death of any cause or the 2016 cutoff. Every observation was staged according to the 7th edition of the AJCC Cancer Staging Manual TNM staging system published in $2010 .^{7}$

\section{Statistical Analyses}

Survival curves were plotted using the Kaplan-Meier method. Univariate and multivariate analyses were performed using a Cox proportional hazards regression model. The following clinicopathologic factors were included in the analyses: age, sex, histologic grade, cell type, therapeutic methods, type of surgery, and T stage. Chi-square and $t$ tests were used to compare differences between categorical and continuous variables, respectively. Cox proportional hazards regression model was used to determine the effects of therapeutic methods and types of surgery on OS. All calculations were performed using SPSS Statistics, version 13 for Windows (SPSS Inc). $P$ values $<.05$ were considered statistically significant.

\section{Results}

Data from 17,954 patients with lung cancer were analyzed, among whom 4,436 had stage T1N2, 8,296 had stage T2N2, and 5,222 had stage T3N2 disease (Table 1).
Mean age was approximately 67 years in each group. The proportion of men increased as the $\mathrm{T}$ stage advanced (T1 stage, $\mathrm{n}=2,082$ [46.93\%]; T2 stage, $\mathrm{n}=4,325$ [52.13\%]; T3 stage, $\mathrm{n}=2,967[56.82 \%])$.

The predominant histologic grade among patients with N2 lung cancer was poorly differentiated (T1 stage, $\mathrm{n}=1,133$ [25.54\%]; T2 stage, $\mathrm{n}=2,658$ [32.04\%]; T3 stage, $n=1,684[32.25 \%])$, followed by moderately differentiated, well differentiated, and undifferentiated. Adenocarcinoma was the predominant histologic cell subtype in the T1N2 stage $(n=1,975 ; 44.52 \%)$ compared with SCC $(\mathrm{n}=775 ; 17.47 \%)$ and others $(\mathrm{n}=1,686 ; 38.01 \%)$. In contrast, SCC was predominant in T3N2 stage $(n=1,924 ; 36.84 \%)$ compared with adenocarcinoma $(n=1,542 ; 29.52 \%)$ and others $(n=1,756$; $33.63 \%)$.

Most patients with N2 lung cancer received only C/T; a substantial proportion received OP plus adjuvant C/T with or without RT $(23.40 \%, 21.47 \%$, and $14.19 \%$ for stages T1N2, T2N2, and T3N2, respectively), and only approximately $3 \%$ received neoadjuvant $\mathrm{C} / \mathrm{T}$ with or without RT followed by OP plus adjuvant $\mathrm{C} / \mathrm{T}$ with or without RT $(2.91 \%, 3.40 \%$, and $3.45 \%$ for stages T1N2, T2N2, and T3N2, respectively). Relatively few patients $(\sim 29.7 \%)$ with $\mathrm{N} 2$ lung cancer received surgical treatment. Lobectomy, the most common surgical type (T1 stage, $\mathrm{n}=1,184$ [26.69\%]; T2 stage, $\mathrm{n}=2,133$ [25.71\%]; T3 stage, $\mathrm{n}=844[16.16 \%]$ ), was performed significantly more often than pneumonectomy and sublobectomy.

The 1-, 3-, and 5-year OS rates were all significantly different among T1N2, T2N2, and T3N2 stages. Figure 1 illustrates the 5-year OS rates for T1N2, T2N2, and T3N2 stages $(27.7 \%, 21.8 \%, 19.9 \%$, respectively; $P<.0001)$.

Table 2 lists survival rates based on therapeutic methods, which were defined as OP only (group 1); OP plus adjuvant $\mathrm{C} / \mathrm{T}$ with or without RT (group 2); neoadjuvant $\mathrm{C} / \mathrm{T}$ with or without $\mathrm{RT}$ followed by $\mathrm{OP}$ plus adjuvant $\mathrm{C} / \mathrm{T}$ with or without RT (group 3); only CCRT (group 4); and C/T and others (group 5). Groups 2 and 3 were not significantly different and had better 5 -year OS than groups 1 and 4 . This result indicates the importance of OP and adjuvant therapy in treating cT1-3N2 lung cancer. Multimodal therapy produced better 5-year OS than single therapy.

Figure 2 shows OS rates based on the different therapeutic methods in all patients with N2 lung cancer, whereas Figures 2B, C, and D display OS rates for those with T1N2, T2N2, and T3N2 disease, respectively. Each figure illustrates significant differences between therapeutic methods $(P<.0001)$.

Figure 3 illustrates 5-year OS rates based on different types of surgery. Lobectomy produced the best outcome 


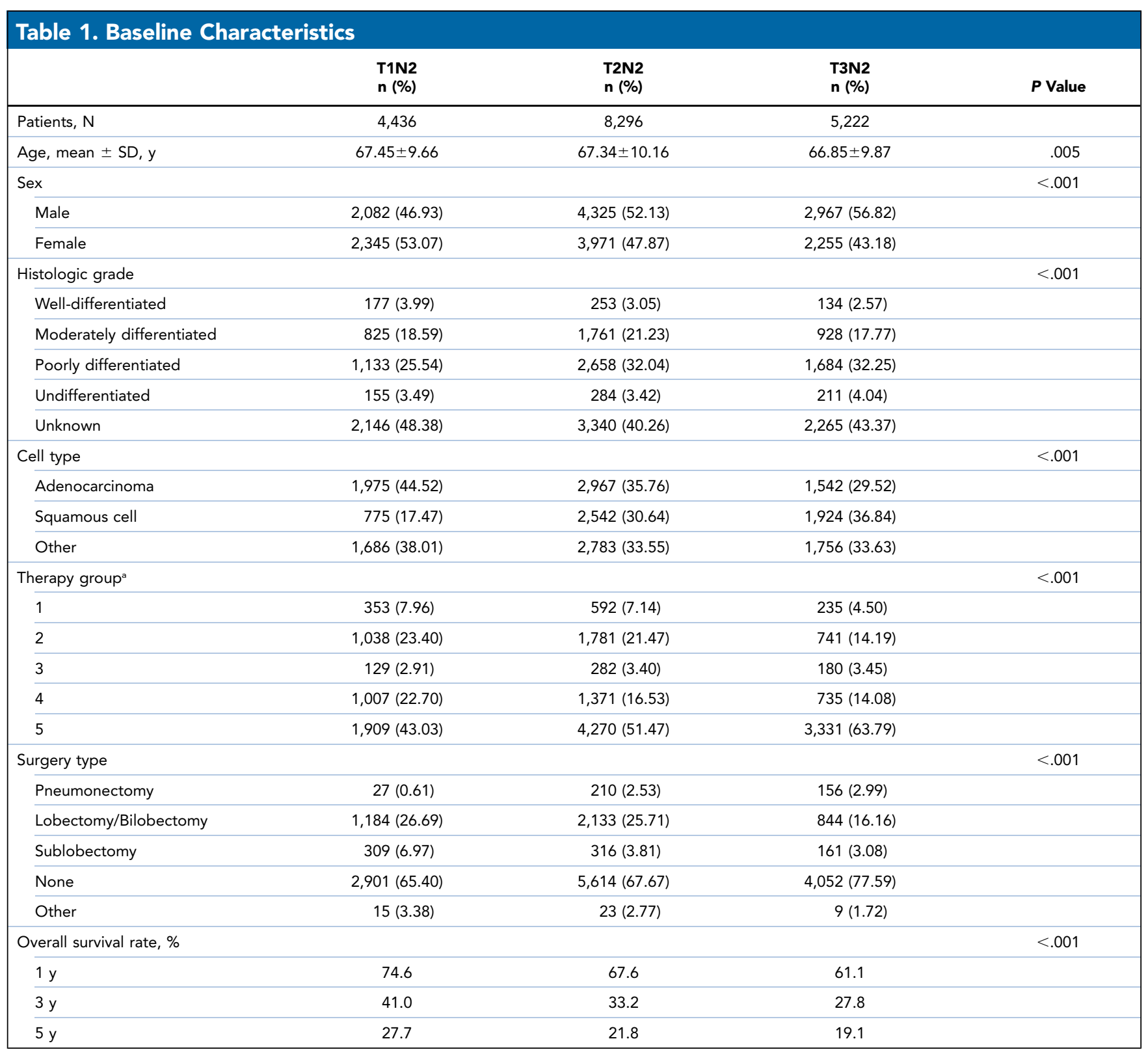

Abbreviations: CCRT, concurrent chemoradiotherapy; $\mathrm{C} / \mathrm{T}$, chemotherapy; OP, operation; $\mathrm{RT}$, radiotherapy.

aGroup 1: OP only; Group 2: OP + adjuvant $\mathrm{C} / \mathrm{T} \pm \mathrm{RT}$; Group 3: neoadjuvant $\mathrm{C} / \mathrm{T} \pm \mathrm{RT}+\mathrm{OP}+\operatorname{adjuvant} \mathrm{C} / \mathrm{T} \pm \mathrm{RT}$; Group 4: CCRT; Group 5: C/T and others.

in patients with cT1N2, cT2N2, and cT3N2 lung cancer $(P<.0001)$. Pneumonectomy was associated with a slightly better outcome than sublobectomy or no surgery, which may indicate that a clear resection margin is more important than lung function preservation.

Both univariate and multivariate Cox regression models were analyzed (Table 3). Univariate and multivariate analyses both showed that young age, female sex, well-differentiated histologic grade, adenocarcinoma cell type, therapy groups 2 and 3, lobectomy, and T1 stage were statistically significantly associated with better 5-year OS rates.

\section{Discussion}

Findings of this retrospective investigation of treatment modalities for patients with lung cancer suggest that multimodal therapies (neoadjuvant $\mathrm{C} / \mathrm{T}$ with or without RT followed by OP plus adjuvant C/T with or without RT, and OP plus adjuvant $\mathrm{C} / \mathrm{T}$ with or without $\mathrm{RT}$ ) tend to be associated with better 5-year OS in patients with cT1-3 N2 lung cancer than OP alone and CCRT.

With regard to induction therapy, Roth et $\mathrm{al}^{6} \mathrm{com}$ pared the treatment strategies of induction C/T plus surgery versus surgery alone and found significantly better outcomes in the combined modality group (2- and 3-year 


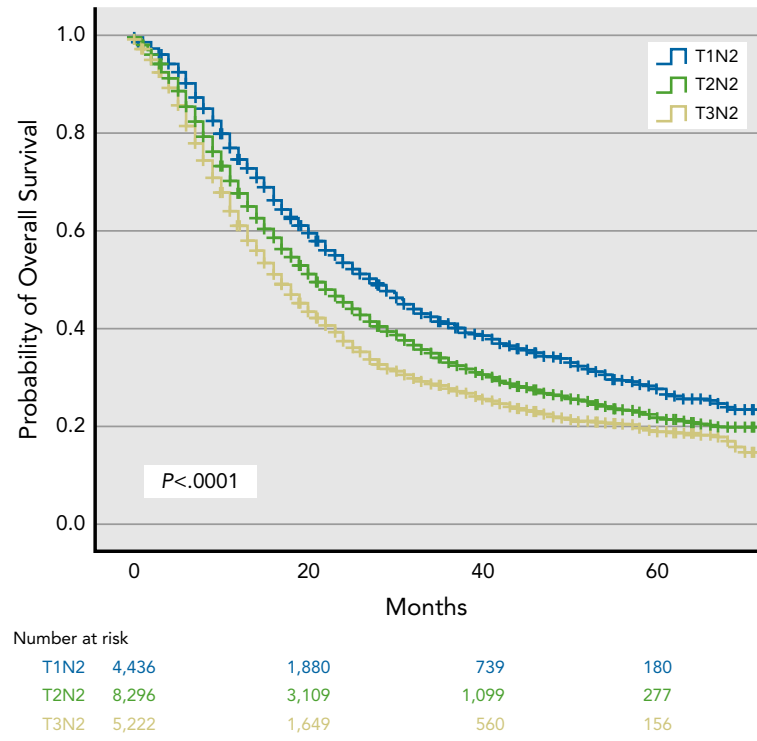

Figure 1. Kaplan-Meier survival curves of different $T$ stages for all patients with N2 lung cancer.

OS rates of $60 \%$ and $56 \%$ vs $25 \%$ and $15 \%$, respectively). In contrast, another randomized trial that analyzed 62 patients with stage IIIA/N2 non-small cell lung cancer (NSCLC) - half receiving induction $\mathrm{C} / \mathrm{T}$ plus $\mathrm{OP}$ and half receiving OP alone ${ }^{8}$-found that 1 - and 3-year OS rates were similar in both groups $(68 \%$ and $23 \%$ for combined modality vs $65 \%$ and $26 \%$ for OP alone, respectively). The investigators concluded that the result may be explained by limited statistical power. Moreover, induction therapy was reported to have no excessive operative risk (morbidity or mortality) compared with direct surgical treatment. ${ }^{9}$ Our findings revealed that induction therapy provided similar 5-year OS rates in N2 disease (comparing groups 2 and 3), which may be explained by the small number of patients in group 3. Use of induction therapy before surgical treatment requires further evaluation.

Several studies have compared induction CRT and induction $\mathrm{C} / \mathrm{T}$ alone, with most revealing that induction CRT only increased pathologic response and mediastinal downstaging but did not improve OS or disease-free survival rates. ${ }^{10-12}$ In particular, the large SAKK and WJTOG9903 trials concluded that CRT does not provide better progression-free survival (PFS) and OS than C/T alone. ${ }^{13,14}$ Based on these concordant findings, we categorized induction CRT and induction $\mathrm{C} / \mathrm{T}$ into the same group (group 3) for comparison.

Some studies have questioned the role of surgery in the treatment of patients with N2 lung cancer. EORTC 08941 concluded that, after induction C/T, surgical resection did not improve OS or PFS compared with RT. ${ }^{15}$ The ESPATUE trial concluded that, after induction $\mathrm{C} / \mathrm{T}$, there was no evidence of better survival in the surgical

\begin{tabular}{|lcccccc|}
\hline \multicolumn{7}{c}{ Table 2. Five-Year Overall Survival Rates } \\
\hline \multirow{7}{*}{ Clinical Stage } & $\mathbf{1}$ & $\mathbf{2}$ & $\mathbf{3}$ & $\mathbf{4}$ & $\mathbf{5}$ & \multirow{P}{*}{ P Value } \\
\cline { 2 - 6 } & 0.344 & 0.420 & 0.413 & 0.203 & 0.000 & $<.001$ \\
\hline T1N2 & 0.297 & 0.395 & 0.377 & 0.143 & 0.117 & $<.001$ \\
\hline T2N2 & 0.237 & 0.297 & 0.274 & 0.156 & 0.081 & $<.001$ \\
\hline T3N2 & & & &
\end{tabular}

Abbreviations: CCRT, concurrent chemoradiotherapy; $\mathrm{C} / \mathrm{T}$, chemotherapy; $\mathrm{OP}$ operation; RT, radiotherapy.

aGroup 1: OP only; Group 2: OP + adjuvant C/T \pm RT; Group 3: neoadjuvant $\mathrm{C} / \mathrm{T} \pm \mathrm{RT}+\mathrm{OP}+$ adjuvant $\mathrm{C} / \mathrm{T} \pm \mathrm{RT}$; Group 4: CCRT; Group 5: $\mathrm{C} / \mathrm{T}$ and others.

group compared with the CRT boost group. ${ }^{16}$ However, the Intergroup 0139 trial showed that a subgroup of patients who underwent lobectomy after CRT showed improved OS rates compared with those who underwent CRT alone. ${ }^{17}$ Our study indicates that patients who underwent OP had better 5-year OS than those who underwent only CCRT or $\mathrm{C} / \mathrm{T}$. We suggest that $\mathrm{OP}$ is essential for patients with cT1-3N2 lung cancer.

The type of surgery that is selected is an important factor. The GLCCG trial analyzed 524 patients with operable stage III NSCLC and concluded that, after induction with CRT, pneumonectomy should be avoided. ${ }^{18}$ The Intergroup 0139 trial showed that OS improved for an OP group that underwent lobectomy but not pneumonectomy. ${ }^{17} \mathrm{~A}$ recent study, published in 2019, reached a similar conclusion and suggested that patients with N2-positive NSCLC treated with fewer pneumonectomy resections experienced favorable OS. ${ }^{10}$ Our findings indicated that patients who received surgical treatment had better OS than those who did not. Lobectomy provided the best 5-year OS, followed by pneumonectomy, sublobectomy, and no surgery.

With regard to adjuvant therapy, a meta-analysis integrated data from 47 trials of stage III resectable NSCLC and reported 2 conclusions. ${ }^{19}$ First, adding $\mathrm{C} / \mathrm{T}$ after surgery increased 5-year survival (from 60\% to 64\%). Second, OP plus adjuvant $\mathrm{C} / \mathrm{T}$ and RT was associated with a higher 5 -year OS rate than OP plus C/T only ( $33 \%$ vs $29 \%$, respectively). Adjuvant therapy played an important role in treating patients with resectable lung cancer, and adding RT provided better outcomes than $\mathrm{C} / \mathrm{T}$ alone. In our study, we categorized adjuvant $\mathrm{C} / \mathrm{T}$ and $\mathrm{RT}$ into one group and also showed that adding adjuvant therapy provided a significantly better 5-year OS than OP alone.

When treating inoperable tumors, CCRT followed by durvalumab is recommended. Durvalumab was introduced as a lung cancer treatment in 2015. The phase III PACIFIC trial was the largest and most recent investigation of durvalumab in patients with NSCLC. ${ }^{20}$ It enrolled 709 patients with stage III NSCLC with unresectable tumors who did not experience disease progression after CCRT. A dose of $10 \mathrm{mg}$ per kilogram of 
A

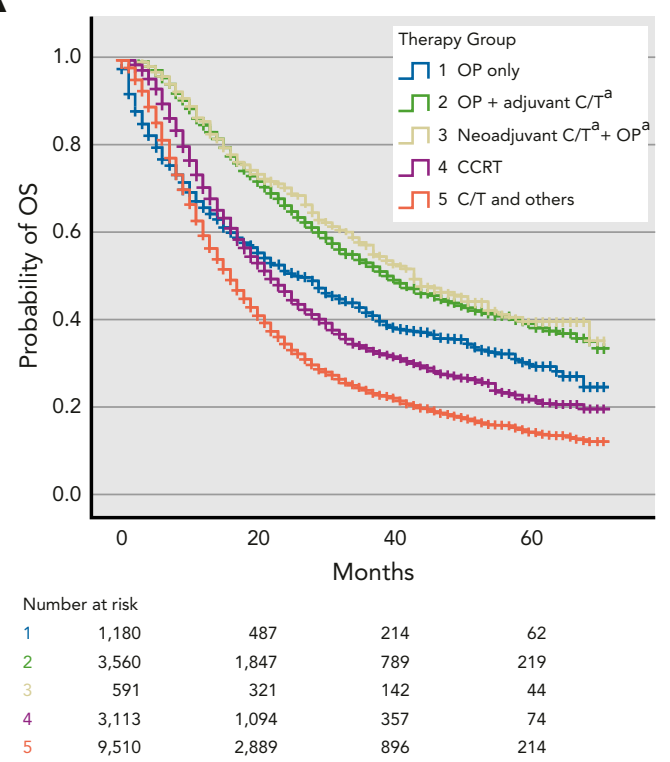

C

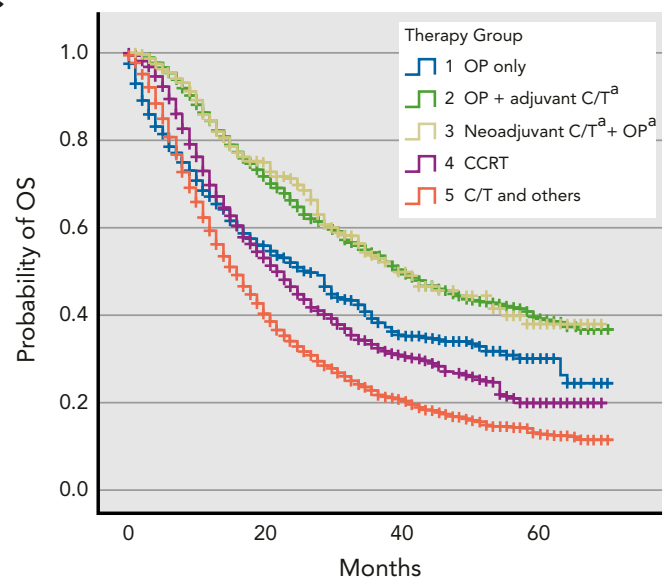

B

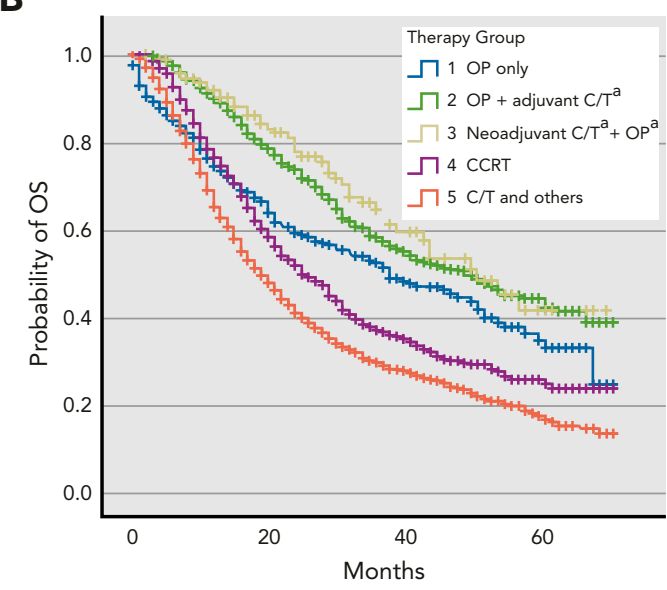

Number at risk

$\begin{array}{rrrrr}1 & 353 & 170 & 79 & 22 \\ 2 & 1,038 & 602 & 268 & 69 \\ 3 & 129 & 81 & 35 & 10 \\ 4 & 1,007 & 376 & 132 & 28 \\ 5 & 1,909 & 651 & 225 & 51\end{array}$

D

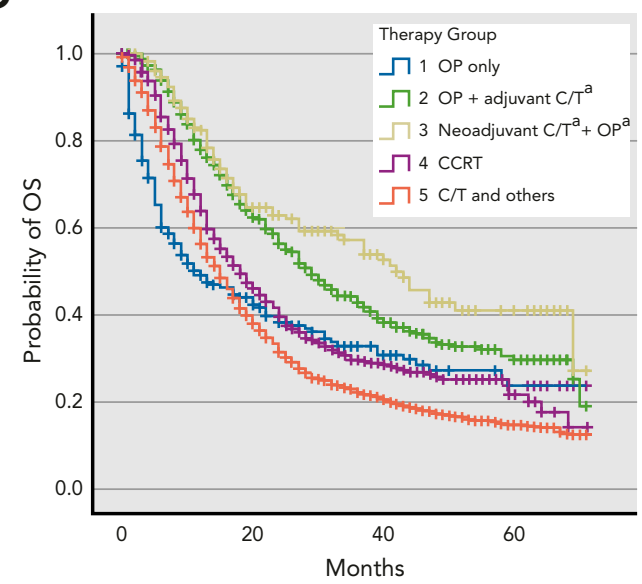

Figure 2. Kaplan-Meier survival curves based on different therapeutic methods for patients with clinical stage (A) T1-3N2, (B) T1N2, (C) T2N2, and (D) T3N2 lung cancer $(P<.0001$ for all).

Abbreviations: CCRT, concurrent chemoradiotherapy; C/T, chemotherapy; OP, operation; OS, overall survival; RT, radiotherapy.

aWith or without RT.

body weight every 2 weeks for 12 months showed a significant benefit compared with placebo in the 24 -month OS rate ( $66.3 \%$ vs $55.6 \%$, respectively), median PFS duration (17.2 vs 5.6 months, respectively), and median time to death or distant metastasis (28.3 vs 16.2 months, respectively). Thus, the standard of care for patients with unresectable stage III NSCLC includes treatment with durvalumab after CCRT.

The well-known RADIANT and SELECT trials indicated that patients with resected EGFR-mutant stage
IB-IIIA NSCLC could benefit from adjuvant EGFR tyrosine kinase inhibitor treatment. As a result, immunotherapy and targeted therapies have become more popular. ${ }^{21,22}$ The new paradigms in stage IIIA (N2) disease have been studied recently. For example, the ADJUVANT/ CTONG1104 trial showed that adjuvant gefitinib led to significantly longer disease-free survival than adjuvant $\mathrm{C} / \mathrm{T}$ in patients with completely resected stage II-IIIA (N1-N2) EGFR-mutant NSCLC. ${ }^{23}$ Additionally, the RTOG 0839 trial evaluated panitumumab at a dose of $2.5 \mathrm{mg} / \mathrm{kg}$ 
A

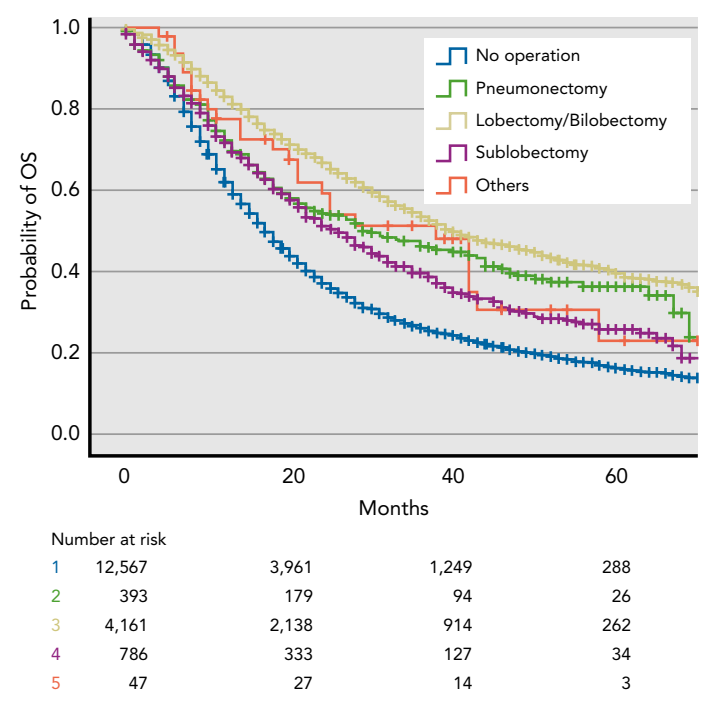

C

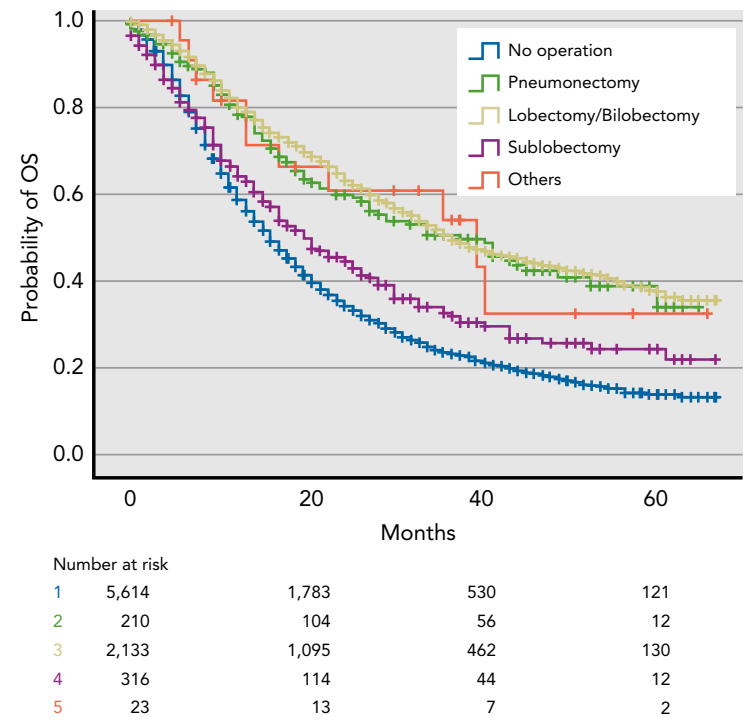

B

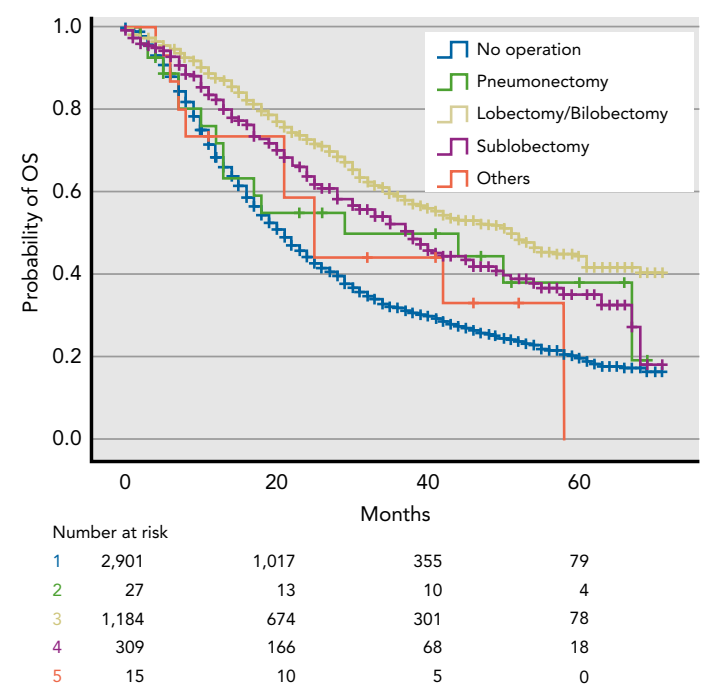

D

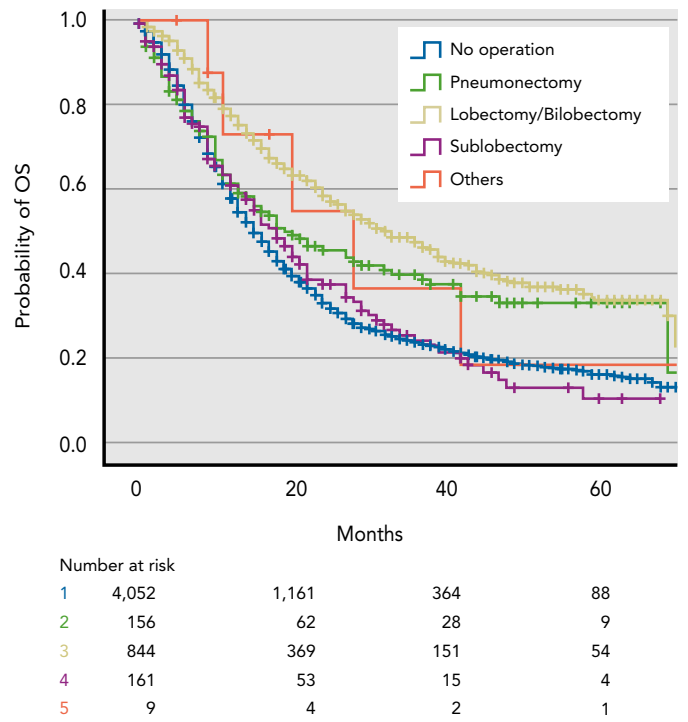

Figure 3. Kaplan-Meier survival curves based on different types of surgery for patients with clinical stage (A) T1-3N2, (B) T1N2, (C) T2N2, and (D) T3N2 lung cancer $(P<.0001$ for all).

Abbreviation: OS, overall survival.

weekly for 6 weeks with neoadjuvant CRT, followed by surgery and consolidation $\mathrm{C} / \mathrm{T}^{24}$; however, there was an unexpectedly high mortality rate in the panitumumab arm. The combination of immunotherapy and targeted therapies with traditional therapy for stage IIIA (N2) disease must be studied further.

A strength of our study was the inclusion of a large number of patients from multiple centers, which should provide accurate statistical analyses. In addition, we compared common therapeutic methods and different surgical types. However, our study had several limitations. First, the retrospective design may have contributed to selection bias, which could affect the results. Second, the study period was limited to data available between 2010 and 2015 only, which could have produced inaccurate 5 -year OS due to inadequate observations. Third, the SEER database did not indicate whether a patient was treated at an academic medical center or a low-volume center. Several studies have indicated that high-volume centers provide better outcomes than low-volume centers for patients with stage III NSCLC. ${ }^{25-27}$ Fourth, currently, the evaluation of lymph nodes for NSCLC is difficult. In stage IIIA (N2) disease, the lymph node station, histologic type, and numbers of metastatic and 
Table 3. Univariate and Multivariate Analysis of Overall Survival

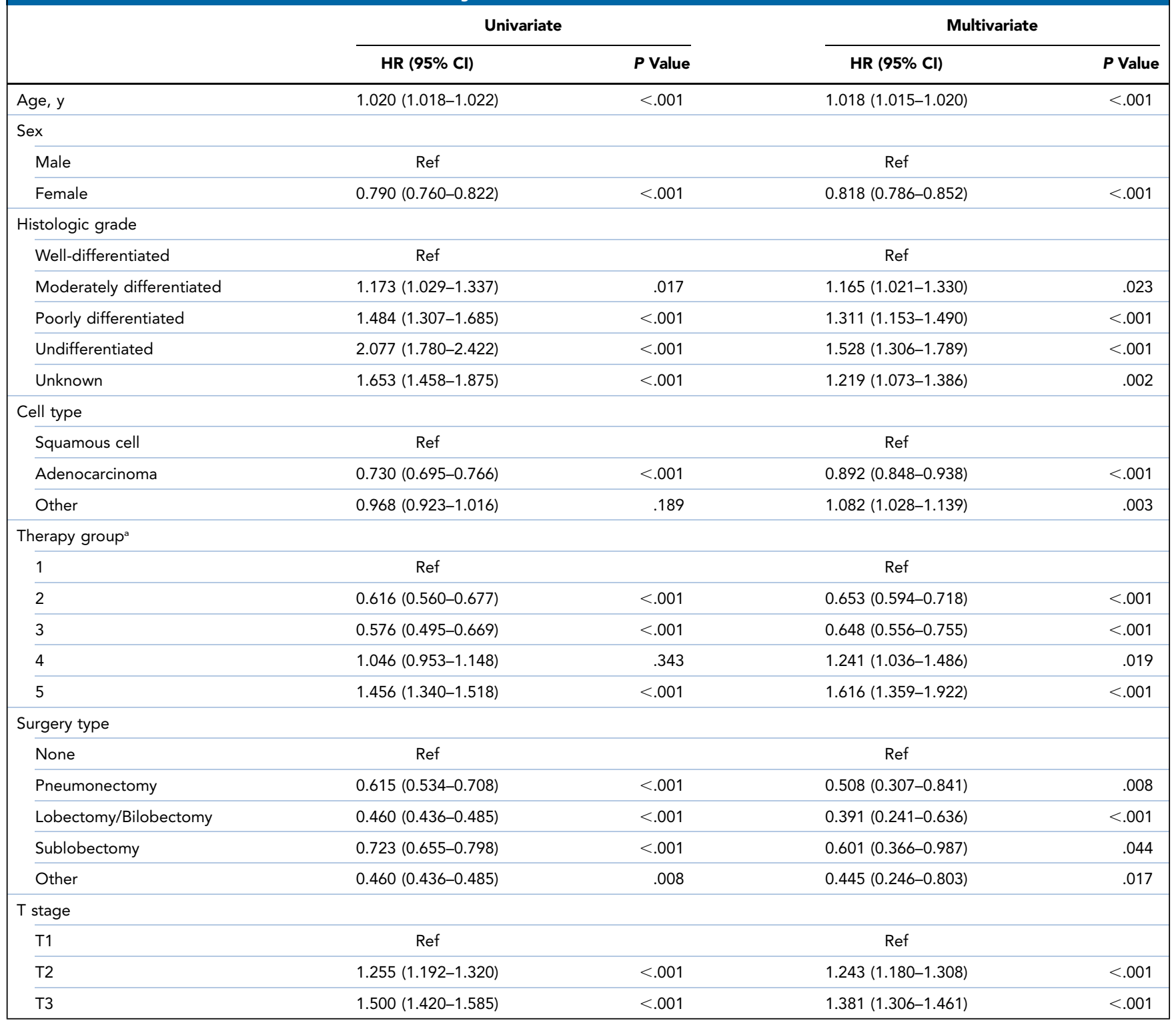

Abbreviations: CCRT, concurrent chemoradiotherapy; $\mathrm{C} / \mathrm{T}$, chemotherapy; $\mathrm{HR}$, hazard ratio; OP, operation; RT, radiotherapy.

aGroup 1: OP only; Group 2: OP + adjuvant $\mathrm{C} / \mathrm{T} \pm \mathrm{RT}$; Group 3: neoadjuvant $\mathrm{C} / \mathrm{T} \pm \mathrm{RT}+\mathrm{OP}+\operatorname{adjuvant} \mathrm{C} / \mathrm{T} \pm \mathrm{RT}$; Group 4: CCRT; Group 5: C/T and others.

skip lymph nodes can lead to different outcomes. ${ }^{28-30}$ NCCN Clinical Practice Guidelines in Oncology have not included these as prognostic factors, and the SEER database did not provide lymph node information. Despite these limitations, our findings contribute to the identification of an optimal treatment strategy for patients with N2 lung cancer.

\section{Conclusions}

In patients with cT1-3N2 lung cancer, multimodal treatments tended to provide better 5-year OS than OP alone and CCRT. In addition, lobectomy was associated with better survival than other operative methods.

Submitted May 6, 2019; accepted for publication August 30, 2019

Author contributions: Study concept and design: Y.F. Cheng, Wang. Technical design and analysis of data: Hung, Chen, C.Y. Cheng. Data acquisition, interpretation, and analysis: C.H. Lin, S.H. Lin.

Disclosures: The authors have not received any financial consideration from any person or organization to support the preparation, analysis, results, or discussion of this article.

Correspondence: Bing-Yen Wang, MD, PhD, Division of Thoracic Surgery, Department of Surgery, Changhua Christian Hospital, 135 Nanxiao Street, Changhua City, Changhua County 500, Taiwan. Email: thomas21917@hotmail.com 


\section{References}

1. Torre LA, Bray F, Siegel RL, et al. Global cancer statistics, 2012. CA Cancer J Clin 2015;65:87-108.

2. Youlden DR, Cramb SM, Baade PD. The international epidemiology of lung cancer: geographical distribution and secular trends. J Thorac Oncol 2008;3:819-831.

3. Siegel RL, Miller KD, Jemal A. Cancer statistics, 2016. CA Cancer J Clin 2016;66:7-30

4. Farjah F, Flum DR, Varghese TK Jr, et al. Surgeon specialty and long-term survival after pulmonary resection for lung cancer. Ann Thorac Surg 2009; 87:995-1004, discussion 1005-1006.

5. Rocco G, Nason K, Brunelli A, et al. Management of stage IIIA (N2) nonsmall cell lung cancer: a transatlantic perspective. Ann Thorac Surg 2016; 101:1247-1250.

6. Roth JA, Fossella F, Komaki $R$, et al. A randomized trial comparing perioperative chemotherapy and surgery with surgery alone in resectable stage IIIA non-small-cell lung cancer. J Natl Cancer Inst 1994;86:673-680.

7. Edge SB, Byrd DR, Compton CC, et al, eds. AJCC Cancer Staging Manual, 7th ed. New York, NY: Springer; 2010.

8. Nagai $K$, Tsuchiya $R$, Mori $T$, et al. A randomized trial comparing induction chemotherapy followed by surgery with surgery alone for patients with stage IIIA N2 non-small cell lung cancer (JCOG 9209). J Thorac Cardiovasc Surg 2003;125:254-260.

9. Evans NR III, Li S, Wright CD, et al. The impact of induction therapy on morbidity and operative mortality after resection of primary lung cancer. J Thorac Cardiovasc Surg 2010;139:991-996.e1-e2.

10. Spicer JD, Shewale JB, Nelson DB, et al. Multimodality therapy for N2 non-small cell lung cancer: an evolving paradigm. Ann Thorac Surg 2019; 107:277-284

11. Shah AA, Berry MF, Tzao C, et al. Induction chemoradiation is not superior to induction chemotherapy alone in stage IIIA lung cancer. Ann Thorac Surg 2012;93:1807-1812.

12. Higgins K, Chino JP, Marks LB, et al. Preoperative chemotherapy versus preoperative chemoradiotherapy for stage III (N2) non-small-cell lung cancer. Int J Radiat Oncol Biol Phys 2009;75:1462-1467.

13. Pless M, Stupp R, Ris HB, et al. Induction chemoradiation in stage IIIA/N2 non-small-cell lung cancer: a phase 3 randomised trial. Lancet 2015;386: 1049-1056.

14. Katakami N, Tada H, Mitsudomi $\mathrm{T}$, et al. A phase 3 study of induction treatment with concurrent chemoradiotherapy versus chemotherapy before surgery in patients with pathologically confirmed N2 stage IIIA nonsmall cell lung cancer (WJTOG9903). Cancer 2012;118:6126-6135.

15. Vansteenkiste J, Betticher D, Eberhardt W, et al. Randomized controlled trial of resection versus radiotherapy after induction chemotherapy in stage IIIA-N2 non-small cell lung cancer. J Thorac Oncol 2007;2:684-685.

16. Stephens RJ, Girling DJ, Hopwood P, et al. A randomised controlled trial of pre-operative chemotherapy followed, if feasible, by resection versus radiotherapy in patients with inoperable stage $\mathrm{T} 3, \mathrm{~N} 1, \mathrm{M} 0$ or T1-3, N2, M0 non-small cell lung cancer. Lung Cancer 2005:49:395-400.
17. Albain KS, Swann RS, Rusch VW, et al. Radiotherapy plus chemotherapy with or without surgical resection for stage III non-small-cell lung cancer: a phase III randomised controlled trial. Lancet 2009;374:379-386.

18. Thomas $M$, Rübe $C$, Hoffknecht $P$, et al. Effect of preoperative chemoradiation in addition to preoperative chemotherapy: a randomised trial in stage III non-small-cell lung cancer. Lancet Oncol 2008;9:636-648.

19. NSCLC Meta-analyses Collaborative Group. Adjuvant chemotherapy, with or without postoperative radiotherapy, in operable non-small-cell lung cancer: two meta-analyses of individual patient data. Lancet 2010 375:1267-1277.

20. Antonia SJ, Villegas A, Daniel D, et al. Overall survival with durvalumab after chemoradiotherapy in stage III NSCLC. N Engl J Med 2018;379: 2342-2350.

21. Kelly K, Altorki NK, Eberhardt WE, et al. Adjuvant erlotinib versus placebo in patients with stage IB-IIIA non-small-cell lung cancer (RADIANT): a randomized, double-blind, phase III trial. J Clin Oncol 2015;33: 4007-4014.

22. Pennell NA, Neal JW, Chaft JE, et al. SELECT: a phase II trial of adjuvant erlotinib in patients with resected epidermal growth factor receptormutant non-small-cell lung cancer. J Clin Oncol 2019;37:97-104.

23. Zhong WZ, Wang $Q$, Mao WM, et al. Gefitinib versus vinorelbine plus cisplatin as adjuvant treatment for stage II-IIIA (N1-N2) EGFR-mutant NSCLC (ADJUVANT/CTONG1104): a randomised, open-label, phase 3 study. Lancet Oncol 2018;19:139-148.

24. Edelman MJ, Hu C, Le QT, et al. Randomized phase II study of preoperative chemoradiotherapy \pm panitumumab followed by consolidation chemotherapy in potentially operable locally advanced (stage IIla, N2+) non-small cell lung cancer: NRG Oncology RTOG 0839. J Thorac Onco 2017;12:1413-1420.

25. Eaton BR, Pugh SL, Bradley JD, et al. Institutional enrollment and survival among NSCLC patients receiving chemoradiation: NRG Oncology Radiation Therapy Oncology Group (RTOG) 0617. J Natl Cancer Inst 2016; 108:djw034.

26. Wang $E H$, Rutter $C E$, Corso $C D$, et al. Patients selected for definitive concurrent chemoradiation at high-volume facilities achieve improved survival in stage III non-small-cell lung cancer. J Thorac Oncol 2015;10: 937-943.

27. Samson P, Patel A, Crabtree TD, et al. Multidisciplinary treatment for stage IIIA non-small cell lung cancer: does institution type matter? Ann Thorac Surg 2015;100:1773-1779.

28. Katsumata S, Aokage K, Ishii G, et al. Prognostic impact of the number of metastatic lymph nodes on the eighth edition of the TNM classification of NSCLC. J Thorac Oncol 2019;14:1408-1418.

29. Wang L, Zhan C, Gu J, et al. Role of skip mediastinal lymph node metastasis for patients with resectable non-small-cell lung cancer: a propensity score matching analysis. Clin Lung Cancer 2019;20:e346-355.

30. Hu XF, Duan L, Jiang GN, et al. Surgery following neoadjuvant chemotherapy for non-small-cell lung cancer patients with unexpected persistent pathological N2 disease. Mol Clin Oncol 2016;4:261-267. 